\title{
Diffusion-weighted MRI improves response assessment after definitive radiotherapy in patients with NSCLC
}

\author{
Jagoda Philippe ${ }^{*^{*}}$ D, Fleckenstein Jochen², Sonnhoff Mathias², Schneider Günther ${ }^{1}$, Ruebe Christian²,
} Buecker Arno ${ }^{1}$ and Stroeder Jonas ${ }^{1}$

\begin{abstract}
Background: Computed tomography $(\mathrm{CT})$ is the standard procedure for follow-up of non-small-cell lung cancer (NSCLC) after radiochemotherapy. CT has difficulties differentiating between tumor, atelectasis and radiation induced lung toxicity (RILT). Diffusion-weighted imaging (DWI) may enable a more accurate detection of vital tumor tissue. The aim of this study was to determine the diagnostic value of MRI versus CT in the follow-up of NSCLC.

Methods: Twelve patients with NSCLC stages I-III scheduled for radiochemotherapy were enrolled in this prospective study. CT with i.v. contrast agent and non enhanced MRI were performed before and 3, 6 and 12 months after treatment. Standardized ROls were used to determine the apparent diffusion weighted coefficient (ADC) within the tumor. Tumor size was assessed by the longest longitudinal diameter (LD) and tumor volume on DWI and CT. RILT was assessed on a 4-point-score in breath-triggered T2-TSE and CT.

Results: There was no significant difference regarding LD and tumor volume between MRI and CT ( $p \geq 0.6221$, respectively $p \geq 0.25$ ). Evaluation of RILT showed a very high correlation between MRI and CT at $3(r=0.8750)$ and 12 months ( $r=0.903$ ). Assessment of the ADC values suggested that patients with a good tumor response have higher ADC values than non-responders.

Conclusions: DWI is equivalent to CT for tumor volume determination in patients with NSCLC during follow up. The extent of RILT can be reliably determined by MRI. DWI could become a beneficial method to assess tumor response more accurately. ADC values may be useful as a prognostic marker.
\end{abstract}

Keywords: Tomography, Spiral computed, Magnetic resonance imaging, Functional magnetic resonance imaging, Radiotherapy, Image-guided, Radiation pneumonitis, Lung neoplasms

\section{Background}

Stereotactic body radiotherapy (SBRT) has a high curative potential and offers excellent local tumor control in inoperable early stage NSCLC [1] while chemoradiotherapy is the standard treatment for inoperable, locally advanced NSCLC [2].

\footnotetext{
* Correspondence: philippe.jagoda@uks.eu

${ }^{1}$ Clinic for Diagnostic and Interventional Radiology, Saarland University Medical Center, Kirrberger Str. 1, 66421 Homburg, Saar, Germany Full list of author information is available at the end of the article
}

In stage III substantial improvements have been achieved during the past two decades with 5-year survival rates now surpassing 30\% [3]. Yet, even with effective newer standards in radiotherapy (the integration of FDG-PET/CT based treatment planning, the application of involved-field target volume concepts to spare organs at risk and the use of intensity modulated radiotherapy [IMRT]) local tumor control still remains unsatisfactory. An actuarial locoregional recurrence rate of $40 \%$ after 3 years was reported by Garg et al. [4], in another

C C The Author(s). 2021 Open Access This article is licensed under a Creative Commons Attribution 4.0 International License, which permits use, sharing, adaptation, distribution and reproduction in any medium or format, as long as you give appropriate credit to the original author(s) and the source, provide a link to the Creative Commons licence, and indicate if changes were made. The images or other third party material in this article are included in the article's Creative Commons licence, unless indicated otherwise in a credit line to the material. If material is not included in the article's Creative Commons licence and your intended use is not permitted by statutory regulation or exceeds the permitted use, you will need to obtain permission directly from the copyright holder. To view a copy of this licence, visit http://creativecommons.org/licenses/by/4.0/ The Creative Commons Public Domain Dedication waiver (http://creativecommons.org/publicdomain/zero/1.0/) applies to the data made available in this article, unless otherwise stated in a credit line to the data. 
retrospective report of Kandi et al. 67 of 137 patients $(48,9 \%)$ experienced a locoregional recurrence after chemoradiotherapy [5]. An early diagnosis of recurrence or detecting a lack of tumor response is essential to provide the patient with an alternative treatment approach such as immunotherapy. For this reason, surveillance is important for monitoring the primary tumor site and detecting metastases.

Several oncological societies such as the National Comprehensive Cancer Network (NCCN) and the American College of Chest Physicians (ACCP) have generated guidelines for post-treatment surveillance imaging of NSCLC [6, 7]. The recommendations stated in these guidelines are based on low-grade evidence [8] and most existing studies address post-operative rather than postradiation follow up.

The currently available guidelines recommend computed tomography (CT) of the chest as standard modality every 6-12 month after treatment of lung cancer for the first 2 years. CT is an established method that is easily available almost anywhere and will quickly generate high-resolution images with isotropic voxels that allow reconstructions in all spatial planes. However, follow up imaging by $\mathrm{CT}$ following radiation therapy can be challenging to interpret, because of radiation induced lung disease (RILT) or formation of tumor-atelectasiscomplex [9]. In addition, iodine-containing contrast agent, which in turn carries risks, is obligatory in order to better evaluate mediastinal and hilar structures.

Thus, an examination which masters the differentiation of tumor atelectasis complex as well as distinguishing RILT versus recurrence would be advantageous. A suitable method that could fulfill this requirement is thoracic magnetic resonance tomography (MRI). In particular, diffusion weighted imaging (DWI) turns out to be promising. DWI is a technique which measures the movement of water molecules known as "brownian motion" using magnetic field gradients. Lesions with high cell density, like tumor tissue, appear bright in DWI imaging due to decreased diffusion. Recently published studies already pointed out the benefit of DWI in chest MRI for differential diagnosis of pulmonary nodules [10] as well as for initial staging of lung cancer and evaluation of lymph node status in particular [11, 12]. Furthermore, a study group could already show a good correspondence of initial tumor volume determination when comparing PET-CT and DWI [13]. However, there is only limited data about the value of DWI in the follow-up assessment of NSCLC after radiochemotherapy. In terms of tumor response, there is an ongoing debate about the predictive value of DWI in regard to apparent diffusion coefficient (ADC).

The aim of our study was to determine the diagnostic value of MRI, especially DWI, versus CT in the follow- up of NSCLC patients after radiochemotherapy. In addition to the assessment of the primary tumor course, a focus was placed on the evaluation of RILT.

\section{Methods}

Study population and inclusion criteria

Eligible patients were at least 18 years old and had a histological diagnosis of non-metastasized NSCLC (UICC stages I-III), had no contraindications to MRI, did not receive any previous antitumoral therapy and were allocated to receive-depending on tumor stage-either SBRT or definitive radiotherapy with concurrent chemotherapy. Written informed consent was obtained from all patients prior to study inclusion. This prospective study was conducted in accordance with the Helsinki declaration and was approved by the local ethics committee (blinded for review).

\section{Follow-up interval}

The patients received both a planning CT and MRI before the start of radiotherapy. Follow-up CT and MRI were performed at 3, 6 and 12 months after radiotherapy. Follow-up CT examinations were performed in an outpatient setting according to the standard of care. All MRI examinations were performed at the Department of Diagnostic and Interventional Radiology. In order to ensure the best possible comparability of the two modalities, the time interval between the two examinations was kept as small as possible. The median interval between the acquisition of the CT and the MRI was 8 days (range 5-12 days) for the pretherapeutic examinations, 7 days (range 1-34) for the 3-month follow-up, 7 days (range 1-33) for the 6-month follow-up and 4days (range 0-13) for the 12-month follow-up.

\section{Acquisition of $\mathrm{CT}$ and MRI images}

Patients received their initial planning CT in the Department of Radiotherapy and Radiation Oncology. The planning CT was a Philips BigBoreTM $120 \mathrm{kV}$ scanner (Philips Medical Systems, Amsterdam, The Netherlands). Patients received iodinated intravenous contrast medium adapted to body weight. The slice thickness was $3 \mathrm{~mm}$, images were acquired during free shallow breathing. Only patients with stage I NSCLC designated for SBRT received an additional 4D-CT. Outpatient follow-up CTs were performed in inspiratory breath hold and after body weight-adapted administration of iodinated contrast medium.

All MRI examinations were performed using the same 1.5 T MRI scanner (Magnetom Aera, Siemens, Erlangen, Germany). A Half Fourier Acquisition Single Shot Turbo Spin Echo (HASTE) sequence $(\mathrm{TE}=91 \mathrm{~ms}, \mathrm{TR}=1000$ ms, Flip-angle $=125^{\circ}$, averages $=1$, slice thickness $5 \mathrm{~mm}$, FOV $=285 \times 380 \mathrm{~mm}$, matrix $=320 \times 192)$ was acquired 
in transverse and coronal planes. To generate diffusionweighted images a single-shot echo planar diffusionweighted sequence with Stejskal-Tanner diffusion encoding scheme using an inversion recovery for fat saturation $(\mathrm{TR}=15,400 \mathrm{~ms}, \mathrm{TE}=75 \mathrm{~ms}, \mathrm{TI}=180 \mathrm{~ms}$, PAT factor of 2, 3-scan trace (averaged), averages $=4$, slice thickness $5 \mathrm{~mm}, \mathrm{FOV}=309 \times 380 \mathrm{~mm}$, matrix $=208 \times$ 128 (interpolated to $208 \times 256$ ), no gap) was acquired. The real voxel size of the sequence was $1.5 \times 3 \times 5 \mathrm{~mm}^{3}$. Two $\mathrm{b}$-values at $\mathrm{b}=0$ and $\mathrm{b}=800 \mathrm{~s} / \mathrm{mm}^{2}$ were acquired. Fusion Images were composed of the HASTE and the DWI. ADC maps and additional high b-value images at $\mathrm{b}=1400 \mathrm{~s} / \mathrm{mm}^{2}$ were calculated automatically by the scanner software, based on linear signal decay. Both HASTE and DWI sequences were acquired with the patient breathing freely; these sequences were subsequently coregistered for image fusion.

To ensure adequate image quality, the DWI sequences were checked by the supervising medical assistant and the physician on duty immediately after their acquisition.

In the event of any imaging artifacts, the DWIsequences were repeated (in altogether three examinations the DWI had to be repeated due to artifacts).

In addition, a respiratory gated T2-weighted sequence $\left(\mathrm{TE}=106 \mathrm{~ms}\right.$, $\mathrm{TR}=3692 \mathrm{~ms}$, Flip- angle $=160^{\circ}$, averages $=2,3 \mathrm{~mm}$ slice thickness, FOV $=277 \times 370 \mathrm{~mm}$, matrix $=384 \times 202$ ) was acquired in a transverse plane.

\section{Assessment of the tumor size, ADC values and RILT}

Generally, all indicated tumor measurements relate to the primary tumor. The tumor size was evaluated according to RECIST 1.1. Thus, the longest longitudinal diameter (LD) of the lesion was measured and classified accordingly to its treatment response into "stable disease" (SD), "partial response" (PR), "progressive disease" $(\mathrm{PD})$ and complete remission (CR). Depending on the course of the disease either baseline or nadir was used to compare and classify the tumor response. Furthermore, the extent of the primary cancer was determined by volumetric measurement. The tumor volume in its course was, in contrast to RECIST 1.1, always related to the baseline.

The examinations were evaluated by two observers with a work experience of 9 and 22 years in consensus. To generate the CT-derived tumor volume both soft tissue and lung window were regarded and the tumor was finally delineated in the lung window. For RECISTevaluation, the longest longitudinal diameter of the primary cancer in lung window was measured. The delineation of the diffusion-weighted tumor volume was performed on diffusion weighted MR image sets and the measurement of the longest longitudinal diameter was also carried out on DWI. The performing observers were blinded for the corresponding CT tumor diameter and volume. The DWI-based contours were delineated visually and secondarily checked and-if necessary-adjusted for anatomical plausibility on the corresponding T2weighted sequence.

DWI was also studied as a functional marker. The ADC maps were used as functional values. The tumor region with the lowest $\mathrm{ADC}$ was identified by consensus by the two radiologists and a standardized ROI of 100 $\mathrm{mm}^{2}$ was placed in this area. The ROIs had to be located completely within the tumor and were standardized to size for better comparability.

Consequently, the ROI had to be adjusted in some cases so that the average size of the ROI was 99.13 $\mathrm{mm}^{2} \pm 6.91$. T2-weighted MR images were used as a reference, to avoid the inclusion of necrotic areas. The mean and standard deviation as well as minimum and maximum values of the ROIs were recorded.

Each study was examined for morphological signs of RILT. A 5-point score established for CT examinations [14] was adapted to a 4-point score to assess RILT $(0=$ no radiation pneumonitis, $1=$ reticular lung parenchyma changes, 2 = inhomogeneous consolidation, $3=$ homogeneous consolidations). The CT examinations were evaluated in the lung window and the MRI examinations on respiratory-triggered T2-TSE images by the two observer pairs. The classification into the respective score was made by consensus. Furthermore, the interobserver variability of the longest tumor diameter and the RILT score was determined.

The evaluation of the longest longitudinal diameters, ADC values and RILT was done on a PACS workstation (SECTRA IDS 7 workstation, Sectra AB, Linkoeping, Sweden). Volume analysis was performed using a medical imaging software (Osirix MD 6.0, Pixmeo Sarl, Switzerland).

\section{Statistics}

Statistical analysis was performed using GraphPad Prism (Prism $^{\circ} 8$ for Mac, Version 8.00, GraphPad Software Inc., San Diego, CA, USA). Values are displayed as median and range (min. to max.) because normal distribution was not assumed. Nonparametric data was further analyzed with a Wilcoxon matched-pairs signed rank test. Correlation for non-normal distributed values was tested using the Spearman correlation test $\left(R_{S}\right)$. For comparison of the 4 ADC timepoints a mixed-effect analysis was performed with a Tukey test afterwards. Interobserver variability was evaluated using weighted kappa for ordinal variables. The assessment of agreement was made according to Landis et al. [15]. For continuous data, reliability was assessed using the intraclass correlation coefficient (ICC), which is commonly considered 
very good when greater than 0.90 [16]. The significance level was defined as $p<0.05$.

\section{Results}

\section{Patients' and treatment characteristics}

Twelve patients ( 3 female, 9 male) with histologically proven NSCLC were included in the study between July 2013 and November 2015. The median age was 68.5 years (range $42-79$ years). One patient had two synchronous lesions of NSCLC (in both upper lobes), which were evaluated separately. TNM stages, histologies, UICC-stages, the localization of the primary tumor and follow-up classification according to RECI ST 1.1 are shown in Table 1. The distribution of UICC-stages and histology was as follows: IA $(n=3)$, IIB $(n=1)$, IIIA $(\mathrm{n}=3)$ and IIIB $(\mathrm{n}=3)$ and IIIC $(n=$ $2)$; squamous cell cancer $(n=8)$, adenocarcinoma $(n=$ 4 whereof two were present in one patient) and not otherwise specified' $(\mathrm{n}=1)$. In 3 patients with early stages (one of them with two lesions) stereotactic body radiotherapy was indicated. These patients received $4 \times 12$ Gy (equaling a biologically effective dose for an $\alpha / \beta$ - value of $10\left[\mathrm{BED}_{10}\right]$ of $\left.105.6 \mathrm{~Gy}\right)$, prescribed to the surrounding $80 \%$ isodose line of the PTV. Definitive radiochemotherapy was planned for all other patients with locally advanced stages using intensity modulated radiotherapy (IMRT) with a median isocenter dose of $60 \mathrm{~Gy}$ (range 58-66.6; single doses 1.8-2.0 Gy). For all patients (median PTV size of $484 \mathrm{~mL}$ [24.5-1407]), dosimetric parameters for organs at risk were as follows (given as median values): mean lung dose 14.9 Gy (4-20.8), V20 (total lung) 26\% (3-30.2), V5 (total lung) 63.5\% (17-88), mean esophageal dose 27.7 Gy (2.1-37.1), mean heart dose 6.85 Gy (0.1-33.5), spinal cord - maximum dose 37.3 Gy (1.8-42.8).

\section{Assessment of tumor size using RECIST 1.1/ LD}

Figure 1 shows tumor response in CT and DWI over the three follow-up examinations.

\section{3-month follow-up}

After 3 months, $13 \mathrm{CT}$ and $12 \mathrm{DWI}$ data sets were available. A total of 12 data pairs were comparable. In one

Table 1 Representation of the TNM stage, histology, the UICC stage, the localization of the primary tumor as well as the classification of the follow-up investigations according to RECIST 1.1. Note - PEC = squamous cell carcinoma, Adeno = Adeno carcinoma, $\mathrm{SD}=$ stable disease, $\mathrm{PD}=$ Progessive Disease, $\mathrm{PR}=$ Partial Remission, $\mathrm{n} . \mathrm{d}$. = not detectable, Pneumonitis = not detectable because of severe pneumonitis, m.a. = missed appointment

\begin{tabular}{|c|c|c|c|c|c|c|c|c|c|c|c|}
\hline Case & TNM & Histology & Primarius & UICC & $\begin{array}{l}3 \text { month } \\
\text { CT }\end{array}$ & $\begin{array}{l}3 \text { month } \\
\text { DWI }\end{array}$ & $\begin{array}{l}6 \text { months } \\
\text { CT }\end{array}$ & $\begin{array}{l}6 \text { months } \\
\text { DWI }\end{array}$ & $\begin{array}{l}12 \text { months } \\
\mathrm{CT}\end{array}$ & $\begin{array}{l}12 \text { months } \\
\mathrm{CT}\end{array}$ & $\begin{array}{l}12 \text { months } \\
\text { DWI }\end{array}$ \\
\hline 1 & $\begin{array}{l}\text { T4 N2 } \\
\text { M0 }\end{array}$ & PEC & $\begin{array}{l}\text { right upper } \\
\text { lobe }\end{array}$ & III B & SD & SD & SD & m.a. & deceased & deceased & deceased \\
\hline 2 & $\begin{array}{l}\text { T3 N0 } \\
\text { MO }\end{array}$ & PEC & middle lobe & II B & $P R$ & SD & $\mathrm{SD}$ & CR & n.d. fibrosis & Pneumonitis & $C R$ \\
\hline 3 & $\begin{array}{l}\text { T1b N0 } \\
\text { M0 }\end{array}$ & PEC & $\begin{array}{l}\text { left lower } \\
\text { lobe }\end{array}$ & I A & $\mathrm{SD}$ & PR & PD & PD & PD & PD & PD \\
\hline 4 & $\begin{array}{l}\text { T4 N3 } \\
\text { M0 }\end{array}$ & PEC & left centrally & III C & PD & PD & deceased & deceased & deceased & deceased & deceased \\
\hline 5 & $\begin{array}{l}\text { T4 N2 } \\
\text { M0 }\end{array}$ & PEC & $\begin{array}{l}\text { right upper } \\
\text { lobe }\end{array}$ & III B & $P R$ & $P R$ & $\mathrm{SD}$ & PD & n.d. fibrosis & Pneumonitis & m.a. \\
\hline 6 & $\begin{array}{l}\text { T4 N3 } \\
\text { M0 }\end{array}$ & Adeno & $\begin{array}{l}\text { left upper } \\
\text { lobe }\end{array}$ & III C & $P R$ & $P R$ & resection & resection & resection & resection & resection \\
\hline 7 & $\begin{array}{l}\text { T2a N2 } \\
\text { M0 }\end{array}$ & PEC & $\begin{array}{l}\text { right lower } \\
\text { lobe }\end{array}$ & III A & $P R$ & PR & Pneumonitis & CR & n.d. fibrosis & Pneumonitis & $C R$ \\
\hline 8 & $\begin{array}{l}\text { T1b N0 } \\
\text { M0 }\end{array}$ & - & $\begin{array}{l}\text { left upper } \\
\text { lobe }\end{array}$ & IA & $P R$ & $\mathrm{SD}$ & $\mathrm{SD}$ & PR & deceased & deceased & deceased \\
\hline 9 & $\begin{array}{l}\text { T4 N2 } \\
\text { M0 }\end{array}$ & Adeno & $\begin{array}{l}\text { right upper } \\
\text { lobe }\end{array}$ & III B & $P R$ & $P R$ & Pneumonitis & PR & n.d. fibrosis & Pneumonitis & PR \\
\hline 10 & $\begin{array}{l}\text { T1b N0 } \\
\text { M0 }\end{array}$ & Adeno & $\begin{array}{l}\text { right upper } \\
\text { lobe }\end{array}$ & $\mid A$ & $\mathrm{SD}$ & $\mathrm{SD}$ & $\mathrm{SD}$ & PR & m.a. & m.a. & m.a. \\
\hline 10 & $\begin{array}{l}\text { T1b N0 } \\
\text { M0 }\end{array}$ & Adeno & $\begin{array}{l}\text { left upper } \\
\text { lobe }\end{array}$ & $\mid A$ & SD & SD & Pneumonitis & $P R$ & m.a. & m.a. & m.a. \\
\hline 11 & $\begin{array}{l}\text { T1a N2 } \\
\text { M0 }\end{array}$ & PEC & $\begin{array}{l}\text { right } \\
\text { centrally }\end{array}$ & III A & $P R$ & $P R$ & $P R$ & n.d. & $\mathrm{SD}$ & SD & PR \\
\hline 12 & $\begin{array}{l}\text { T2a N2 } \\
\text { M0 }\end{array}$ & PEC & left centrally & III A & $P R$ & $P R$ & SD & PR & SD & SD & $P R$ \\
\hline
\end{tabular}




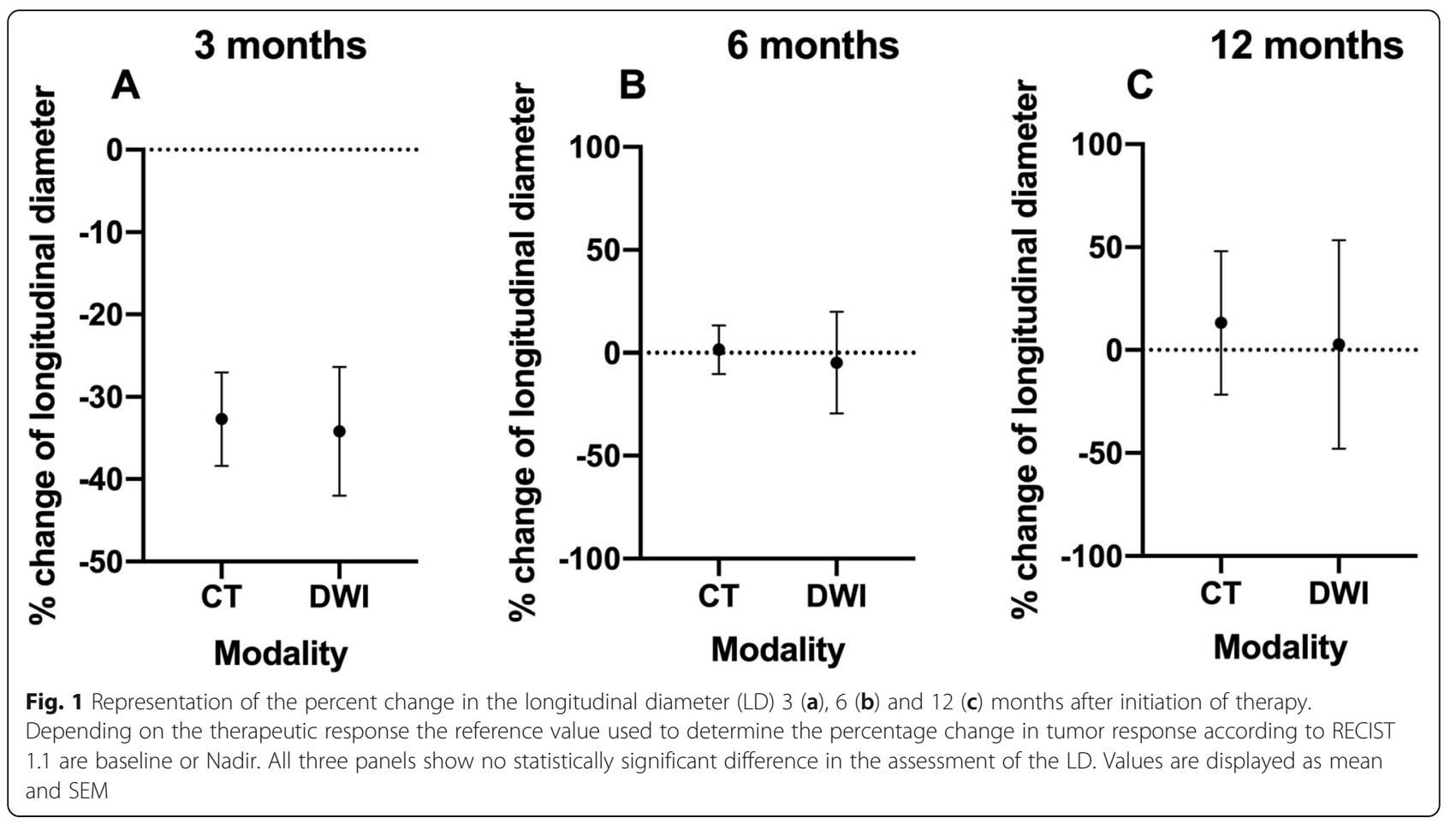

patient the tumor could no longer be clearly identified by DWI; however, in this case, there was no difference in tumor response regarding RECIST 1.1 due to newly occurring distant metastasis. When assessing tumor response, there were 10 matches between CT and DWI as follows: PR $(n=6), \operatorname{SD}(n=3)$ and PD $(n=1)$. Two cases were discrepant, as CT classified them as PR and DWI as SD. However, in both cases of DWI only two percentage points were missing in order to classify them also as PR. A single reverse case in which DWI classified tumor response as PR and CT classified it as SD occurred.

The median percentage size changes of the tumor showed no significant difference between CT $(-33.33$ $[-72.0-5.0])$ and DWI $(-31.86[-76.0-13.92) \quad(p=$ $0.6221)$.

\section{6 -month follow-up}

After 6 months, $8 \mathrm{CT}$ and 7 DWI data sets were available. A total of 5 data pairs were comparable. One patient had died of his tumor disease during this follow up interval.

Another patient left the study because of a resectable tumor after treatment initiation.

Another patient with SD on CT follow-up did not turn up for his MRI appointment. In another patient with SD after CT evaluation, the tumor could no longer be delineated on MRI (CR); retrospectively it could be shown that this patient was free from recurrence until the end of the study. Due to a pronounced fibrosis, no evaluation of the tumor in the right lower lobe was possible in one patient on CT. Interestingly, no considerable diffusion restriction could be seen in the corresponding DWI dataset in this area; however, at this time, in contrast to the CT scan, a diffusion-restricted lesion on the right hilar side was detectable; here the patient developed a recurrence (Fig. 2). In addition, DWI was able to classify 2 patients reliably as $\mathrm{PR}$, whilst no tumor evaluation was possible on CT for these patients due to pneumonitis. One patient was scored on CT as a PR, at which time no tumor was assessable in the DWI. One patient had PD on both DWI and CT. Three cases were discrepant, as CT classified them as SD and DWI as PR, whereas the percentages of the two modalities were in part close to the threshold between PR and SD. The CT classified one patient as SD, which the DWI contrarily classified as PD; retrospectively, the patient developed a recurrence and died after the study.

The median percentage size changes of the tumor showed no significant difference between CT (4.23 [45.83-68.95]) and DWI $(-35.15[-48.46-122.8)(p>$ $0.99)$.

\section{2-month follow-up}

After 12 months, $3 \mathrm{CT}$ and 4 DWI data sets were available. A total of 3 data pairs were comparable. In the meantime, altogether three patients had died; two patients due to tumor progress and one patient due to exacerbated COPD. One patient did not show up at the 


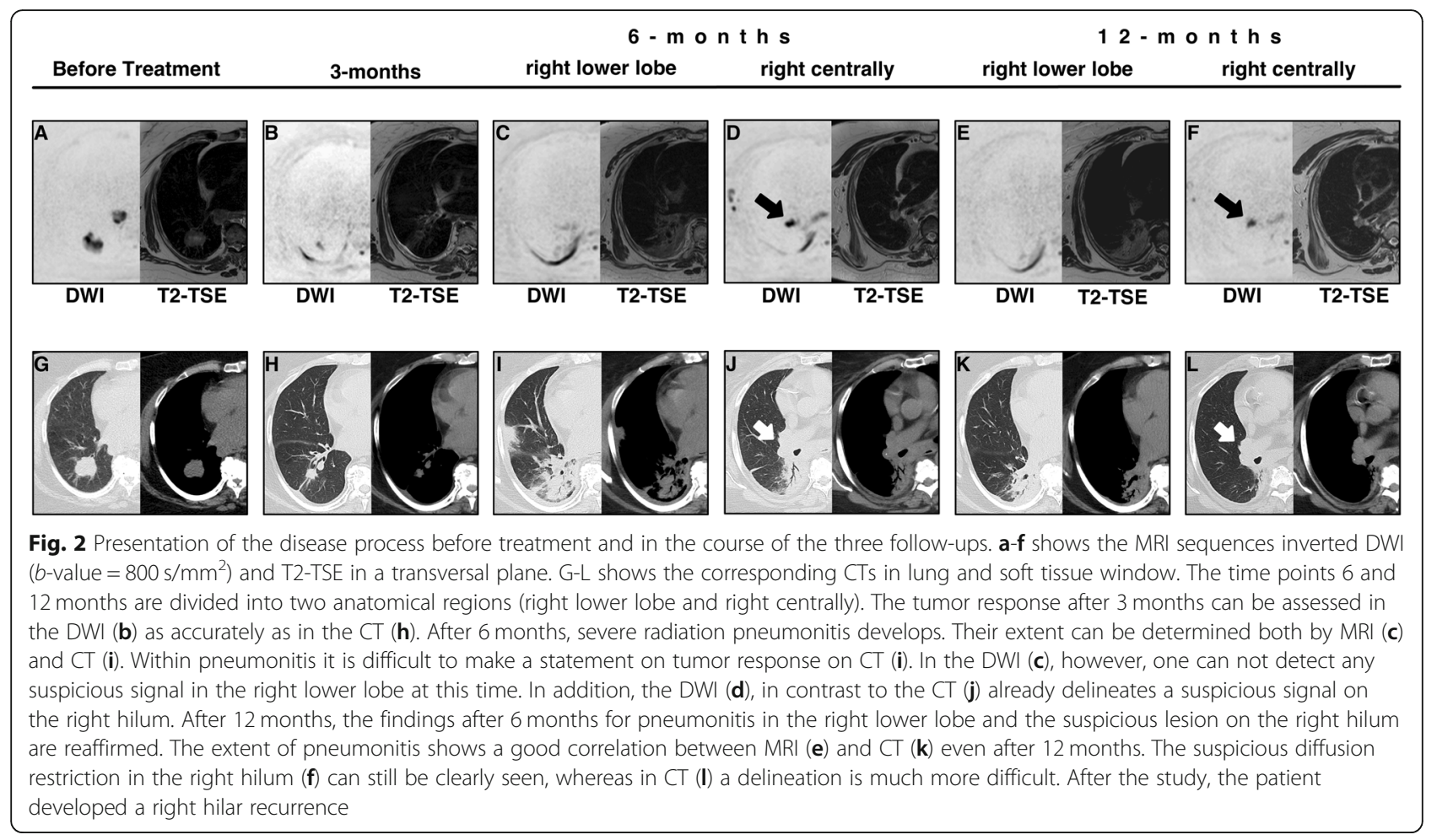

MRI and the CT appointment, another one missed only the MRI appointment. Due to a pronounced fibrosis, no reliable evaluation of the tumor was possible in two patients on CT images, whereas DWI showed no suspect signal in these two patients (CR). The clinical course made it most likely that the diagnosis of $\mathrm{CR}$ was correct.
In another case fibrosis did not allow for a clear statement regarding tumor size on $\mathrm{CT}$ images. The corresponding DWI showed a PR. Again, this diagnosis was confirmed by further follow up. Two patients were scored as SD on CT, whereas being rated as PR by DWI. However, the CT percentage values of those two patients

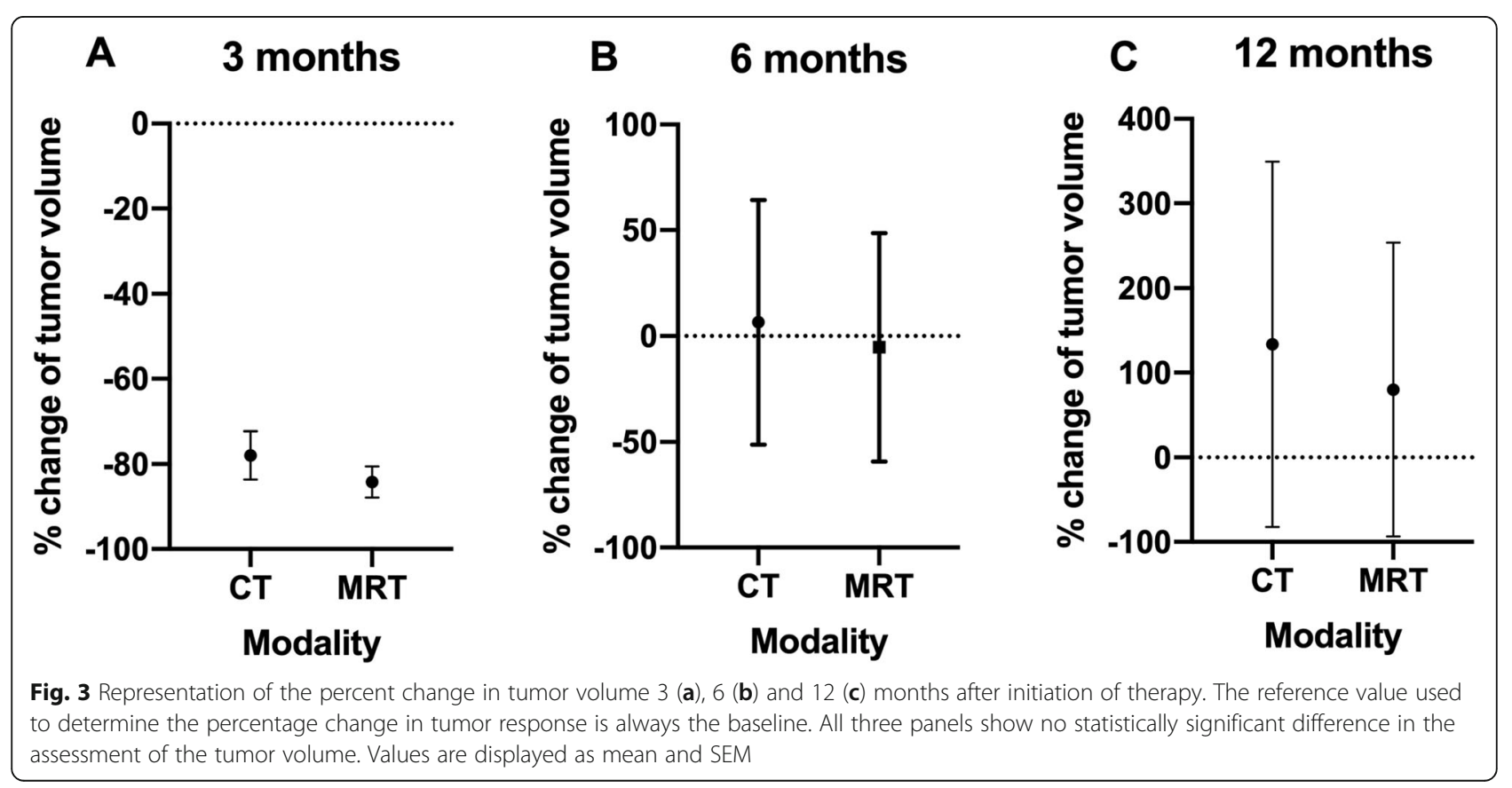


Table 2 Comparison of the absolute percentages of the CT and MRI pneumonitis score at 3, 6 and 12 months after the end of treatment. After 3 and 12 months there was a very good correlation. However, there was only a moderate correlation after 6 months. Overall, the score on MRI tends to be slightly lower than in the corresponding CT. Absolute frequencies are written in parenthesis behind the corresponding percentage value

\begin{tabular}{|c|c|c|c|c|c|c|c|c|c|c|c|c|}
\hline \multicolumn{5}{|c|}{ Pneumonitis Score 3-months follow up } & \multicolumn{4}{|c|}{$\begin{array}{l}\text { Pneumonitis Score 6-months follow } \\
\text { up }\end{array}$} & \multicolumn{4}{|c|}{$\begin{array}{l}\text { Pneumonitis Score 12-months } \\
\text { follow up }\end{array}$} \\
\hline Percentage of grand total & MRI 0 & MRI 1 & MRI 2 & MRI 3 & MRI 0 & MRI 1 & MRI 2 & MRI 3 & MRI 0 & MRI 1 & MRI 2 & MRI 3 \\
\hline СT 0 & $35.71(5)$ & $7.14(1)$ & $0.00(0)$ & $0.00(0)$ & $10.00(1)$ & $0.00(0)$ & $0.00(0)$ & $0.00(0)$ & $33.33(2)$ & $0.00(0)$ & $0.00(0)$ & $0.00(0)$ \\
\hline СТ 1 & $7.14(1)$ & $7.14(1)$ & $0.00(0)$ & $0.00(0)$ & $0.00(0)$ & $10.00(1)$ & $0.00(0)$ & $0.00(0)$ & $0.00(0)$ & $0.00(0)$ & $0.00(0)$ & $0.00(0)$ \\
\hline СТ 2 & $0.00(0)$ & $0.00(0)$ & $14.29(2)$ & $0.00(0)$ & $0.00(0)$ & $10.00(1)$ & $20.00(2)$ & $0.00(0)$ & $0.00(0)$ & $0.00(0)$ & $16.67(1)$ & $0.00(0)$ \\
\hline СТ 3 & $0.00(0)$ & $0.00(0)$ & $28.57(4)$ & $0.00(0)$ & $0.00(0)$ & $20.00(2)$ & $20.00(2)$ & $10.00(1)$ & $0.00(0)$ & $0.00(0)$ & $16.67(1)$ & $33.33(2)$ \\
\hline
\end{tabular}

were again close to the PR threshold. One patient had PD in both CT and DWI.

The median percentage size changes of the tumor showed no significant difference between CT $(-20.00$ $[-23.00-82.75])$ and DWI $(-39.53[-63.56-153.6)(p>$ $0.99)$.

\section{Assessment of tumor volume}

Figure 3 shows the development of tumor volume in CT and DWI over the course of time.

Available CT and DWI data sets as well as comparable data pairs are the same as in the assessment of tumor size using RECIST 1.1/LD.

At $3(p=0.5771), 6(p=0.3125)$ and $12(p=0.2500)$ months, there was no significant difference between CT and DWI in terms of tumor volume. At 3 months, the percentage change in tumor volume was -84.16 [95.74 - -36.43) on CT and - 91.73 [-95.84 - -59.69] on DWI. At 6 months, the percent change in tumor volume was -29.87 [-83.96-228.6) on CT and-34.19 [88.79-204.5] on DWI. And at 12 months, the percent change in tumor volume was -68.29 [-95.88-564.7] on CT and - 89.11 [-98.26-427.1] on DWI.

\section{Assessment of RILT}

Table 2 shows by means of a contingency table the frequency distribution of the pneumonitis scores determined in CT and MRI (T2-TSE).

A total of 13 data pairs were comparable after 3 months. At the 6 months follow up 10 data pairs were comparable and at the 12 months follow up there were 6 data sets available.

The classification of pneumonitis on CT and MRI correlated very well after 3 months $(r=0.88)$. The median score was 1 [0-3] (mean with Std. deviation $1.15 \pm 1.28$ ) on CT and 1 on MRI [0-2] (mean with Std. deviation $0.92 \pm 0.95$ ). After 6 months, there was only a moderate correlation between the two modalities $(r=0.50)$. The median score was 3.0 [0-3] (mean with Std. deviation $2.27 \pm 1.01$ ) on CT and 1.5 on MRI [0-3] (mean with Std. deviation $1.5 \pm 0.85$ ). After 12 months, the correlation was again very good $(\mathrm{r}=0.90)$. The median score was $2.5[0-3]$ (mean with Std. deviation $1.83 \pm$ 1.47) on CT and 2.0 on MRI [0-3] (mean with Std. deviation $1.6 \pm 1.37$ ).

\section{Functional imaging/ADC}

The mean ADC values of each patient in the course of the 4 timepoints are shown in Fig. 4 Panel a. Averaging the ADC values over the 4 time points revealed no statistically significant difference for either the ADC mean $(p=0.15)$ or the ADC maximum $(p=0.16)$ (Fig. 4 Panel $c / d)$. However, an increasing trend could be observed for both values: ADC mean (0-months: $1113 \pm 127.0,3$ months $1500 \pm 144.6,6$ months $1258 \pm 104.8$ and 12 months $1421 \pm 251.5)$ and ADC MAX (0-months: $1533 \pm 133.9,3$ months $1940 \pm 147.9,6$ months $1797 \pm$ 150.9 and 12 months $2100 \pm 477.8$ ). Finally, the patient collective was divided into two groups: one with therapy response (PR) and another one with progressive disease (PD). However, it was not possible to include all patients, since not every patient could be clearly assigned to one of the two groups. The group with response to therapy had a higher ADC value at all times than the group with PD (Fig. 4 Panel b). However, the difference was not statistically significant $(p=0.13)$.

\section{Interobserver variability}

ICC for continuous data, longest diameter, yielded an excellent agreement (ICC $=0.996 ; 95 \%$ CI, 0.990-0.998). Weighted kappa for ordinal data, RILT Score, resulted in a substantial agreement 0.780 (95\% CI, 0.592-0.959).

\section{Discussion}

MRI of the chest had long been technically challenging due to the movement and breathing artifacts of the thoracic organs as well as the susceptibility artifacts caused by the interfaces between different tissues and the overall low proton density of the lung [17].

The continuous technical development in MRI with fast imaging techniques, resulting in protocols with scan 


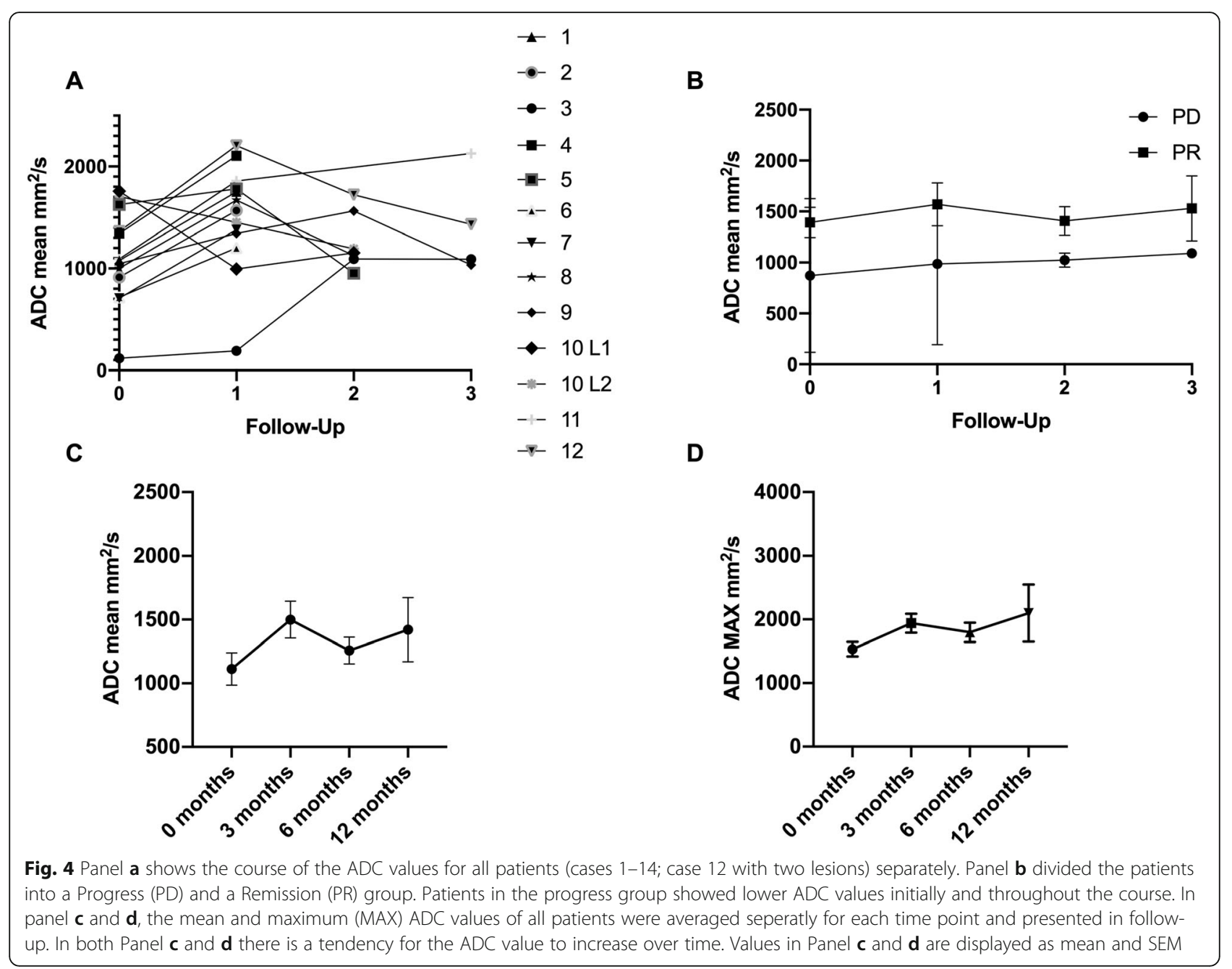

times between 15 and 30 min [18], has made chest MRI an interesting alternative to CT $[19,20]$. Regarding the low evidence of appropriate follow-up examinations in NSCLC, especially after chemoradiation [8], chest MRI might be the future solution. Our study is one of the first longitudinal investigations comparing CT and MRI after chemoradiation of NSCLC regarding morphological and functional parameters.

Prior studies have mainly focused on pretreatment comparison of CT or PET/CT and MRI [13, 18]. Fleckenstein et al. already demonstrated a high level of concordance between the pretreatment tumor volumes of PET-CT and DWI for radiotherapy-planning [13].

FDG-PET/CT has a high diagnostic accuracy in the detection of local tumor recurrences [21] and is often used, when - based on the follow-up CT - a recurrence is suspected [22]. Nevertheless, FDG-uptake is also enhanced in lung regions which show severe RILT. Therefore, the diagnosis of local lung recurrences in areas of RILT might be impaired in such cases. Also, FDG-PET/ $\mathrm{CT}$ is usually more expensive and less available than
DWI and thus contraindicated as a routine measure in the follow-up due to economic and logistic reasons.

We observed no statistically significant difference regarding the percentage change of the longest longitudinal diameter between CT and DWI at 3, 6 and 12 months (Fig. 1). The spread of the measurements within the two modalities can be explained most easily on the different initial tumor sizes and its responses. An inverse tumor response between CT and DWI was not observed. This is also reflected by the classification of the tumor response according to RECIST 1.1. There was a high concordance between DWI and CT regarding therapy response after 3 months resulting in $\mathrm{PR}(n=6), \mathrm{SD}(n=3)$ and $\mathrm{PD}(n=1)$. In the three discrepant cases only a few percentage points were missing for the transition from PR to SD or SD to PR. However, at 6 and 12 months, there is a diminishing correspondence of CT and DWI in terms of tumor response. This observation may have multifactorial causes, such as RILT with limited sensitivity of CT scans, deceased patients and missed appointments. However, in the follow-up at 6 and 12 months in individual cases, the DWI 
detected recurrences earlier than $\mathrm{CT}$ or excluded them with greater certainty (Table 1 [Cases 2 and 7] and Fig. 2). These results are in line with the study published in May 2019 by Usuda et al. [23]. In their study DWI was more accurate than CT in determining a response of recurrent lesions of lung cancer to chemotherapy and/or radiotherapy. Consistent with our study, they concluded that DWI may be able to identify residual cancer, thereby improving specificity and sensitivity.

Conventional response criteria like RECIST 1.1 have some limitations. There is an ongoing debate how accurate a unidimensional measurement can represent the real tumor burden due to varying and often highly irregular tumor shapes. Meanwhile, several studies have demonstrated that volume measurement in lung tumors is more reproducible than size measurement [24, 25]. In addition, the study by Zhang et al. proves that DWI has a more precise delineation of lung cancer while exhibiting higher reproducibility [26]. In our study there was no significant difference between tumor volumes as determined by CT and DWI at any of the three follow-up dates. The tumor volumes in the DWI tended to be slightly smaller than in their CT counterparts. We identified the more precise demarcation of the tumor against atelectatic lung tissue and parenchymal changes in pneumonitis as the major cause for this discrepancy. There is a lack of data comparing CT and DWI tumor volumes in the course of therapy after chemoradiation. A comparable study by Weiss et al. determined significantly larger tumor volumes by $\mathrm{CT}$ as compared to DWI in patients after chemoradiation [27]. However, the results are only partially comparable to the data presented here, since the working group around Weiss et al. chose follow up assessments at 3 and 6 weeks, thus focusing on early changes.

In the future, we will be particularly challenged by the assessment of tumor process under immunotherapy. However, initial studies are already showing the advantages of MRI/DWI in the assessment of tumor response already $[28,29]$.

In addition to assessing tumor response, the applied imaging modality should reliably indicate RILT. RILT typically occurs as early as 4 to 12 weeks after treatment and may transform into radiation fibrosis (which may also occur independently) after 6 months or later [30]. Although clinically debilitating pneumonitis (grade $\geq 3$ ) after radiotherapy develops in less than $10 \%$ of patients [31], imaging in commonly used scores, such as the LENT-SOMA Score from the European Organization for Research and Treatment of Cancer (EORTC) [32], plays a role in diagnosis and therefore therapy. Regarding the evaluation of RILT, some groups use functional investigations in MRI with xenon gas with quite impressive results [33]. Meanwhile, the parenchymal structure of the lungs can be adequately assessed by MRI, as was shown by Sileo et al. in patients suffering from cystic fibrosis [34]. To our knowledge, we hereby present the first investigation to examine the correlation of RILT scores determined by CT and MRI, respectively respiratory gated T2-weighted sequence, over a period of 1 year. At 3 and 12 months a high correlation of the RILT scores was found. However, at the early stage of fibrosis development at 6 months, only a moderate correlation was shown between the two modalities. At this stage, the CT achieved a higher score than the MRI, which may be a hint for its earlier detection of reticular lung parenchyma changes. Overall, however, it can be concluded that respiratory gated T2-weighted sequence can adequately assess the ultrastructure of the lungs in the early and late phase after chemoradiation to diagnose or exclude RILT. Differentiating between treatment effects like RILT or tumor-atelectasis-complex and residual or recurrent tumor, is challenging [35]. Like aforementioned, in some cases of the presented group, by using DWI, as compared to CT, we were able to delineate recurrences earlier and to more reliably rule out recurrence within lung parenchyma altered by RILT (Fig. 2). These results are in line with a study of MunozSchuffenegger et al. in which they could prove that DWI confirmed the suspicion of local recurrence in patients with highly suspicious CT scans [36]. Furthermore, DWI/ADC not only provides these important additional informations but might also be a prognostic factor.

Looking at the individual mean ADC values of patients over the time course, no clear pattern could be observed in our study (Fig. 4a). However, averaging the ADC values of all patients at each time point mean and maximum ADC showed a tendency to increase (Fig. 4c/d). Our results are consistent with prior studies which demonstrated a significant ADC increase after chemoradiation and chemotherapy [27, 37, 38]. Weiss et al. showed that patients with survival $<12$ months had a lower increase in ADC values compared to longer-lived patients [27]. Sampath et al. could demonstrate that an ADC increase of $40 \%$ at 1 month after SBRT for NSCLC is associated with a higher rate of local failure [39]. In contrast, non-responders in the study by Chang et al. had a slight decrease in ADC, whereas responders had a relatively steeper increase of ADC [37]. As opposed to the latter data, after formation of a PD and PR group, we were unable to detect a significant increase or decrease in the mean ADC between the two groups (Fig. 4b), which could be due to the small sample size. However, both the pretherapeutic and the mean $\mathrm{ADC}$ values over the course tend to be lower in non-responders (PD group). In agreement with our findings, Shintani et al. and Iizuka et al. found that low ADC on pre-treatment MRI were associated with local recurrence and poor disease progression [40, 41]. Yet, Ohno et al. reported 
contradictory findings in patients in whom higher ADC on pretreatment MRI were significantly associated with poor prognosis [42].

The discrepancies in the predictive power of the ADC may in part be due to the non-uniform measurement. Depending on the study, the mean, minimum or maximum ADC value is used. Furthermore, until now there is no clear definition of where within the tumor one should place the ADC ROI. Further studies are needed to establish a uniform and reproduceable measurement of the ADC and thus to substantiate its prognostic value.

Beside of all of these capabilities MRI offers in imaging of the NSCLC, the acquisition time of this modality has to be viewed critically especially in comparison with CT. As mentioned in the first section of the Discussion, the MRI protocol takes about 15-30 min (median duration $33 \mathrm{~min}$, range [max to $\mathrm{min}$ ] $19 \mathrm{~min}$ to $1 \mathrm{~h}$ and $13 \mathrm{~min}$ ). Compared to a CT scan of the thorax with an acquisition time of only a few seconds for the actual scan and a few minutes for the entire examination, this is of course a considerable effort, especially for patients with impaired lung function. However, the MRI protocol can certainly be optimized by removing, respectively limiting the time-consuming breath-triggered T2-TSE to the target areas, because acquisition of the whole thorax can take between 15 and 30 min depending on the patient's body height and breathing variability. If assessment of the ultra-structure of the lung is not required, DWI/ ADC in combination with T2-HASTE, both only taking about $5 \mathrm{~min}$ for image acquisition, could be a solution for thorax imaging regarding $\mathrm{T}$ and $\mathrm{N}$ stadium.

Our study had some limitations. First, it is a single center study with a small number of patients. Additionally, some patients did not complete scanning schedule and we can't exclude the possibility that this might have skewed the results.

\section{Conclusion}

In conclusion we present an initial longitudinal study, which demonstrates that after chemoradiation therapy response determined by RECIST 1.1 and tumor volume measurement can be done by DWI yielding similar results to CT. In addition, the presented study is one of the first to describe typical changes of RILT in the early and late phase as diagnosed with MRI as compared to the gold standard of CT. Thus, MRI including DWI, bears a strong potential for improved detection of an inadequate response to radiotherapy or early recurrences. In regard to the potential prognostic value of ADC measurements further investigations are necessary.

\section{Abbreviations}

ACCP: American College of Chest Physicians; ADC: Apparent diffusion coefficient; CR: Complete remission; CT: Computed tomography; DWI: Diffusion weighted imaging; EORTC: European Organization for
Research and Treatment of Cancer; LD: Longitudinal diameter; MRI: Magnetic resonance imaging; NCCN: National Comprehensive Cancer Network; NSCL C: Non small cell lung cancer; PD: Progressive disease; PR: Partial remission; RILT: Radiation induced lung toxicity; SBRT: Stereotactic body radiotherapy; SD: Stable disease; UICC: Union for International Cancer Control

\section{Acknowledgements}

We thank all patients for their willingness to participate in the study. In addition, we are grateful for the collegial and productive cooperation of the two executive departments. We thank Helge Anand Krebs-Fleischmann for his excellent editorial support.

\section{Authors' contributions}

FJ, SG, RC, BA made substantial contributions to conception and design. JP, FJ, MS, JS made substantial contributions to acquisition, analysis and interpretation of the data. JP has drafted and submitted the article. FJ, MS, GS, RC, BA, SJ have revised the article critically and substantially for important intellectual content. JP, FS, MS, GS, RC, BA, SJ have provided final approval of the version to be published. JP, FS, MS, GS, RC, BA, SJ have agreed to be accountable for all aspects of the work in ensuring that questions related to the accuracy or integrity of any part of the work are appropriately investigated and resolved. The author(s) read and approved the final manuscript.

\section{Funding}

The authors state that this work has not received any funding. Open Access funding enabled and organized by Projekt DEAL.

\section{Availability of data and materials}

The datasets used and analysed during the current study are available from the corresponding author on reasonable request.

\section{Ethics approval and consent to participate}

This prospective study was conducted in accordance with the Helsinki declaration and was approved by the local ethics committee (Ethikkommission der Ärztekammer des Saarlandes).

\section{Consent for publication}

Informed consent was obtained from each patient.

\section{Competing interests}

The authors declare that they have no competing interests.

\section{Author details}

${ }^{1}$ Clinic for Diagnostic and Interventional Radiology, Saarland University Medical Center, Kirrberger Str. 1, 66421 Homburg, Saar, Germany.

${ }^{2}$ Department of Radiotherapy and Radiation Oncology, Saarland University Medical Center, Kirrberger Str. Geb. 6.5, 66421 Homburg, Saar, Germany.

Received: 1 June 2020 Accepted: 8 January 2021

Published online: 21 January 2021

References

1. Palma D, Lagerwaard F, Rodrigues G, Haasbeek C, Senan S. Curative treatment of stage i non-small-cell lung cancer in patients with severe COPD: stereotactic radiotherapy outcomes and systematic review. Int J Radiat Oncol Biol Phys. 2012:82:1149-56.

2. McCloskey P, Balduyck B, Van Schil PE, Faivre-Finn C, O'Brien M. Radical treatment of non-small cell lung cancer during the last 5 years. Eur J Cancer. 2013;49:1555-64. Available from:. https://doi.org/10.1016/j.ejca.2012.12.023.

3. Bradley JD, Paulus R, Komaki R, Masters G, Blumenschein G, Schild S, et al. Standard-dose versus high-dose conformal radiotherapy with concurrent and consolidation carboplatin plus paclitaxel with or without cetuximab for patients with stage IIIA or IIIB non-small-cell lung cancer (RTOG 0617): a randomised, two-by-two factorial p. Lancet Oncol. 2015;16:187-99.

4. Garg S, Gielda BT, Kiel K, Turian JV, Fidler MJ, Batus M, et al. Patterns of locoregional failure in stage III non-small cell lung cancer treated with definitive chemoradiation therapy. Pract Radiat Oncol. 2014;4:342-8.

5. Kandi M, Hoffmann L, Moeller DS, Schmidt HH, Knap MM, Hoffmann L, et al. Local failure after radical radiotherapy of non- small cell lung cancer in relation to the planning FDG-PET / CT. Acta Oncol (Madr). 2018;0:1-7. 
Informa UK Limited, trading as Taylor \& Francis Group; Available from. https://doi.org/10.1080/0284186X.2017.1409436.

6. Colt HG, Murgu SD, Korst RJ, Slatore CG, Unger M, Quadrelli S. Follow-up and surveillance of the patient with lung cancer after curative-intent therapy: Diagnosis and management of lung cancer, 3rd ed: American college of chest physicians evidence-based clinical practice guidelines. Chest. 2013;143:e4375-54S. The American College of Chest Physicians; Available from. https://doi.org/10.1378/chest.12-2365.

7. MD FPDRB, FRCP PSP. (NCCN-Guidelines) Non-Small Cell Lung Cancer. PETCT Radiother Treat Plan. 2008:140-52. Available from: https://www. sciencedirect.com/science/article/pii/B9781416032243500116.

8. Dyer BA, Daly ME. Surveillance imaging following definitive radiotherapy for non-small cell lung cancer: what is the clinical impact? Semin Oncol. 2017; 44:303-9.

9. Dunlap NE, Yang W, Mclntosh A, Sheng K, Benedict SH, Read PW, et al. Computed tomography-based anatomic assessment overestimates local tumor recurrence in patients with mass-like consolidation after stereotactic body radiotherapy for early-stage non-small cell lung cancer. Int J Radiat Oncol Biol Phys. 2012;84:1071-7.

10. Satoh S, Kitazume Y, Ohdama S, Kimula Y, Taura S, Endo Y. Can malignant and benign pulmonary nodules be differentiated with diffusion-weighted MRI? Am J Roentgenol. 2008;191(2):464-70.

11. Peerlings J, Troost EGC, Nelemans PJ, Cobben DCP, de Boer JCJ, Hoffmann $A L$, et al. The diagnostic value of MR imaging in determining the lymph node status of patients with non-small cell lung Cancer: a meta-analysis. Radiology. 2016;281(1):86-98.

12. Usuda K, Sagawa M, Motono N, Ueno M, Tanaka M, Machida Y, et al. Advantages of diffusion-weighted imaging over positron emission tomography-computed tomography in assessment of hilar and mediastinal lymph node in lung cancer. Ann Surg Oncol. 2013;20(5):1676-83.

13. Fleckenstein J, Jelden M, Kremp S, Jagoda P, Stroeder J, Khreish F, et al. The Impact of Diffusion-Weighted MRI on the Definition of Gross Tumor Volume in Radiotherapy of Non-Small-Cell Lung Cancer. PLoS One. 2016;11:e0162816.

14. Kimura T, Matsuura K, Murakami Y, Hashimoto Y, Kenjo M, Kaneyasu Y, et al. $\mathrm{CT}$ appearance of radiation injury of the lung and clinical symptoms after stereotactic body radiation therapy (SBRT) for lung cancers: are patients with pulmonary emphysema also candidates for SBRT for lung cancers? Int J Radiat Oncol Biol Phys. 2006;66:483-91.

15. Landis JR, Koch GG. The measurement of observer agreement for categorical data. Biometrics. 1977;33:159-74.

16. Koo TK, Li MY. A guideline of selecting and reporting Intraclass correlation coefficients for reliability research. J Chiropr Med. 2016:15:155-63.

17. Luna A, Sánchez-Gonzalez J, Caro P. Diffusion-weighted imaging of the chest. Magn Reson Imaging Clin N Am. 2011;19:69-94.

18. Kim HS, Lee KS, Ohno Y, Van Beek EJR, Biederer J. PET/CT versus MRI for diagnosis, staging, and follow-up of lung cancer. J Magn Reson Imaging. 2015:42:247-60

19. Biederer J, Ohno Y, Hatabu H, Schiebler ML, van Beek EJR, Vogel-Claussen J, et al. Screening for lung cancer: does MRI have a role? Eur J Radiol. 2017;86: 353-60. Available from:. https://doi.org/10.1016/j.ejrad.2016.09.016.

20. Henzler T, Schmid-Bindert G, Schoenberg SO, Fink C. Diffusion and perfusion MRI of the lung and mediastinum. Eur J Radiol. 2010;76:329-36. Available from:: https://doi.org/10.1016/j.ejrad.2010.05.005.

21. Jimenez-Bonilla JF, Quirce R, Martinez-Rodriguez I, Banzo I, Rubio-Vassallo $A S$, Del Castillo-Matos $R$, et al. Diagnosis of recurrence and assessment of post-recurrence survival in patients with extracranial non-small cell lung cancer evaluated by 18F-FDG PET/CT. Lung Cancer. 2013;81:71-6.

22. Cuaron J, Dunphy M, Rimner A. Role of FDG-PET scans in staging, response assessment, and follow-up care for non-small cell lung cancer. Front Oncol. 2012;2:208.

23. Usuda K, Iwai S, Funasaki A, Sekimura A, Motono N, Matoba M, et al. Diffusion-weighted magnetic resonance imaging is useful for the response evaluation of chemotherapy and/or radiotherapy to recurrent lesions of lung cancer. Transl Oncol. 2019;12:699-704 Available from: http://www.ncbi. nlm.nih.gov/pubmed/30856554.

24. Zhao B, James LP, Moskowitz CS, Guo P, Ginsberg MS, Lefkowitz RA, et al. Evaluating Variability in Tumor Measurements from Same-day Repeat CT Scans of Patients with Non-Small Cell Lung Cancer. Radiology. 2009;252(1):263-72.

25. Nishino M, Guo M, Jackman DM, Di Piro PJ, Yap JT, Ho TK, et al. CT tumor volume measurement in advanced non-small-cell lung cancer. Performance characteristics of an emerging clinical tool. Acad Radiol. 2011;18(1):54-62.
26. Zhang X, Fu Z, Gong G, Wei H, Duan J, Chen Z, et al. Implementation of diffusion-weighted magnetic resonance imaging in target delineation of central lung cancer accompanied with atelectasis in precision radiotherapy. Oncol Lett. 2017;14:2677-82.

27. Weiss E, Ford JC, Olsen KM, Karki K, Saraiya S, Groves R, et al. Apparent diffusion coefficient (ADC) change on repeated diffusion-weighted magnetic resonance imaging during radiochemotherapy for non-small cell lung cancer: a pilot study. Lung Cancer. 2016;96:113-9. Available from:: https://doi.org/10.1016/j.lungcan.2016.04.001.

28. Schiza A, Irenaeus S, Ortiz-Nieto F, Loskog A, Tötterman T, Sundin A, et al. Evaluation of diffusion-weighted MRI and FDG-PET/CT to assess response to AdCD40L treatment in metastatic melanoma patients. Sci Rep. 2019;9:18069.

29. Jiang X, Dudzinski S, Beckermann KE, Young K, McKinley E, McIntyre JO, et al. MRI of tumor T cell infiltration in response to checkpoint inhibitor therapy. J Immunother cancer. 2020;8(1):e000328.

30. Choi YW, Munden RF, Erasmus JJ, Park KJ, Chung WK, et al. Effects of radiation therapy on the lung: radiologic appearances and differential diagnosis. Radiographics. 2004;24:985-97.

31. Linda A, Trovo M, Bradley JD. Radiation injury of the lung after stereotactic body radiation therapy ( SBRT ) for lung cancer : A timeline and pattern of CT changes. Eur J Radiol. 2011;79:147-54.

32. Jain V, Berman AT. Radiation pneumonitis: old problem, new tricks. Cancers. 2018;10:1-16.

33. Ireland RH, Din OS, Swinscoe JA, Woodhouse N, Van Beek EJR, Wild JM, et al. Detection of radiation-induced lung injury in non-small cell lung cancer patients using hyperpolarized helium-3 magnetic resonance imaging. Radiother Oncol. 2010;97:244-8. Available from. https://doi.org/10. 1016/j.radonc.2010.07.013.

34. Sileo C, Corvol H, Boelle PY, Blondiaux E, Clement A, Ducou Le Pointe H. HRCT and MRI of the lung in children with cystic fibrosis: Comparison of different scoring systems. J Cyst Fibros. 2014;13:198-204. Available from. https://doi.org/10.1016/j.jpf.2013.09.003.

35. Yang RM, Li L, Wei XH, Guo YM, Huang YH, Lai LS, et al. Differentiation of central lung Cancer from atelectasis: comparison of diffusion-weighted MRI with PET/CT. PLoS One. 2013;8(4):e60279.

36. Munoz-Schuffenegger P, Kandel S, Alibhai Z, Hope A, Bezjak A, Sun A, et al. A prospective study of magnetic resonance imaging assessment of postradiation changes following stereotactic body radiation therapy for nonsmall cell lung Cancer. Clin Oncol (R Coll Radiol). 2019;31:720-7.

37. Chang $Q$, Wu N, Ouyang H, Huang Y. Diffusion-weighted magnetic resonance imaging of lung cancer at 3.0 T: A preliminary study on monitoring diffusion changes during chemoradiation therapy. Clin Imaging. 2012;36:98103. Available from. https:/doi.org/10.1016/j.clinimag.2011.07.002.

38. Yabuuchi H, Hatakenaka M, Takayama K, Matsuo Y, Sunami S, Kamitani T, et al. Non-small cell lung Cancer: detection of early response to chemotherapy by using contrast-enhanced dynamic and diffusion-weighted MR imaging. Radiology. 2011;261(2):598-604.

39. Sampath S, Rahmanuddin S, Sahoo P, Frankel P, Boswell S, Wong J, et al. Change in apparent diffusion coefficient is associated with local failure after stereotactic body radiation therapy for non-small cell lung Cancer: a prospective clinical trial. Int J Radiat Oncol biol Phys. 2019;105:659-63.

40. Shintani T, Matsuo Y, lizuka Y, Mitsuyoshi T, Umeoka S, Nakamoto Y, et al. Assessment of treatment response after lung stereotactic body radiotherapy using diffusion weighted magnetic resonance imaging and positron emission tomography: a pilot study. Eur J Radiol. 2017;92:58-63. Available from:. https://doi.org/10.1016/j.ejrad.2017.04.022.

41. lizuka Y, Matsuo Y, Umeoka S, Nakamoto Y, Ueki N, Mizowaki T, et al. Prediction of clinical outcome after stereotactic body radiotherapy for non-small cell lung cancer using diffusion-weighted MRI and18F-FDG PET. Eur J Radiol. 2014;83:2087-92. Available from:. https://doi.org/10.1016/j.ejrad.2014.07.018.

42. Ohno Y, Koyama H, Yoshikawa T, Matsumoto K, Aoyama N, Onishi Y, et al. Diffusion-weighted MRI versus 18F-FDG PET/CT: performance as predictors of tumor treatment response and patient survival in patients with non-small cell lung cancer receiving chemoradiotherapy. AJR Am J Roentgenol. 2012; 198:75-82.

\section{Publisher's Note}

Springer Nature remains neutral with regard to jurisdictional claims in published maps and institutional affiliations. 